\title{
Prediction and Management in Energy Harvested Wireless Sensor Nodes
}

\author{
Joaqun Recas Piorno*, Carlo Bergonzini ${ }^{\dagger}$, David Atienza ${ }^{\ddagger}$, Tajana Simunic Rosing $\S$ \\ *DACYA, Complutense University of Madrid, Spain \\ Email: jrecas@fis.ucm.es \\ $\dagger$ DEIS, Universit degli Studi di Bologna, Italy \\ Email: carlo.bergonzini@studio.unibo.it \\ ${ }^{\ddagger}$ Embedded Systems Laboratory (ESL), Ecole Polytechnique Fdrale de Lausanne (EPFL), Switzerland \\ Email: david.atienza@epfl.ch \\ $\S$ Computer Science and Engineering, University of California San Diego, USA \\ Email: tajana@ucsd.edu
}

\begin{abstract}
Solar panels are frequently used in wireless sensor nodes because they can theoretically provide quite a bit of harvested energy. However, they are not a reliable, consistent source of energy because of the Sun's cycles and the everchanging weather conditions. Thus, in this paper we present a fast, efficient and reliable solar prediction algorithm, namely, Weather-Conditioned Moving Average (WCMA) that is capable of exploiting the solar energy more efficiently than state-of-the-art energy prediction algorithms (e.g. Exponential Weighted Moving Average EWMA). In particular, WCMA is able to effectively take into account both the current and past-days weather conditions, obtaining a relative mean error of only $10 \%$. When coupled with energy management algorithm, it can achieve gains of more than $90 \%$ in energy utilization with respect to EWMA under the real working conditions of the Shimmer node, an active sensing platform for structural health monitoring.
\end{abstract}

\section{INTRODUCTION}

Latest battery-powered embedded sensor nodes ([1],[2],[3],[4]), are designed to gather, process and wirelessly transmit data in regular time intervals. These nodes are able to provide significant in-situ processing capabilities of the collected data (e.g., temperature figures, humidity). As a consequence, a whole new set of applications with complex features, such as actuation and data processing, have been developed for these nodes. However, battery lifetime is still a significant limitation. One way to improve this is by using energy harvesting coupled with rechargeable energy storage (e.g. batteries and super capacitors).

Many different types of energy harvesting technologies exist - solar, vibration, wind, piezoelectric, thermoelectric, etc. Solar energy harvesting is by far the most effective [5] for wireless sensor nodes. A great application example is Shimmer [6], a sensor node targeted at structural health monitoring applications (SHM). It uses supercapacitors as energy storage units and a solar panel for energy harvesting. Shimmer evaluates the health of a large structure such as a bridge by sending a wave through it via one PZT device, and sensing a response via another. Fairly significant processing has to be done on the data (over 100 MIPS) to detect if there is damage. Finally it transmits the result. The amount of energy needed to execute these tasks is substantial, thus being able to understand how much energy is currently available, and how much can be harvested in the next time interval is very important.

Online strategies that understand these tradeoffs and can plan in near term how to best spend the energy received via energy harvesting need to be developed. The sensor node should exploit the extra energy available from energy harvesting sources once the batteries or super capacitors are charged up. This energy can be used to achieve higher accuracy by executing additional tasks on the node (such as actuating, sensing, processing, etc). When energy harvesting is either not available or minimal, the sensor node still needs to be able to respond to a minimum amount of outside queries for data (i.e., event-based triggering). Therefore, adaptation is needed as the energy availability and the outside demands change dynamically in time.

In this paper, we propose Weather-Conditioned Moving Average (WCMA), a novel accurate yet very low overhead, solar energy prediction algorithm based on the Exponentially Weighted Moving-Average (EWMA) [7] estimation method. Our new WCMA algorithm can be used to accurately estimate the amount of energy that will be harvested by solar panels in the near future, so that it is possible to deploy power-efficient task management methods on solar energy-harvested wireless sensor nodes.

The rest of the paper is organized as follows. In Section 2 we summarize the related work on energy prediction algorithms. Section 3 outlines our new solar energy harvesting prediction algorithm. Finally, in Section 4 we assess the efficiency of the energy prediction algorithm using an active sensing platform for Structural Health Monitoring, i.e., the Shimmer node.

\section{RELATED WORK}

A frequently used and low cost (in terms of computation need) energy prediction algorithm is Exponentially Weighted Moving-Average (EWMA) [7],[8],[9],[10]. The method is designed to exploit the diurnal cycle in solar energy and to adapt to the seasonal variations. EWMA calculates the value of energy likely to be harvested at a particular time as a 
weighted average of the energy received at the same time over a set of previous days [10]. Although EWMA-based algorithm is accurate for consistent weather conditions, when cloudy and sunny days are mixed, recent days energy values introduce significant prediction errors. Therefore, to prevent this problem, we introduce in this work a new prediction algorithm that not only takes into account the solar conditions at a certain time of the day, but also adjusts the energy intake estimation for the changing weather conditions throughout a day.

Other solar prediction algorithms have been recently proposed, based on mean expected values. In [11], it is shown that the average-daily solar system performance may be calculated from the product of clear-sky solar performance and the average time fraction of clear sky. This approximation greatly simplifies the solar system performance prediction, but does not offer specific energy guarantees at certain daily intervals, not been suitable for short term predictions. Also, [12] introduces a new method for modeling daily sun radiations, based on Takagi-Sugeno fuzzy systems. This method uses a non-linear technique, defined by a set of If-Then rules with linear consequent parts, which establish a local linear input-output relationship between the variables of the model. Then, the parameters of the model are identified using the fuzzy clustering combined to the least square algorithm. This model produces accurate results, but requires a very high computation, making this algorithm not applicable on small wireless sensor nodes as we target in this work.

\section{WCMA ENERGY PREDICTION}

WCMA has its foundations on EWMA for estimating the solar energy entering the system. In contrast to EWMA, it also characterizes the seasonal changes by adapting both the change in the hour of sunrise and sunset, as well as the difference in solar power between seasons. Furthermore, this new algorithm takes into the account weather changes with minimal overhead.

In EWMA, the day is divided on slots and a vector of estimated values for each slot $i$ is stored, i.e., $X(i)$. This equation is used to update the slots, as follows:

$$
X(i)=\alpha \cdot X(i-1)+(1-\alpha) \cdot x(i)
$$

where $x(i)$ denotes the value of real energy observed at the end of the slot $i$ and $\alpha$ is a weighting factor.

Figure 1 shows the actual energy input from the solar panel and the predicted value in five consecutive days, with a mix of sunny and cloudy conditions. In this case, when the sunny and cloudy days alternate, the EWMA produces a significant error in its prediction, due to the high impact of the solar conditions of previous day in the predicted value. To avoid this effect, our new prediction algorithm takes into account not only the solar conditions at a specific time of the day, but also the weather conditions in the current day. This is especially important in frequently changing weather conditions, for example, we observed that the energy harvested during cloudy days was less than half of that gathered during sunny days.

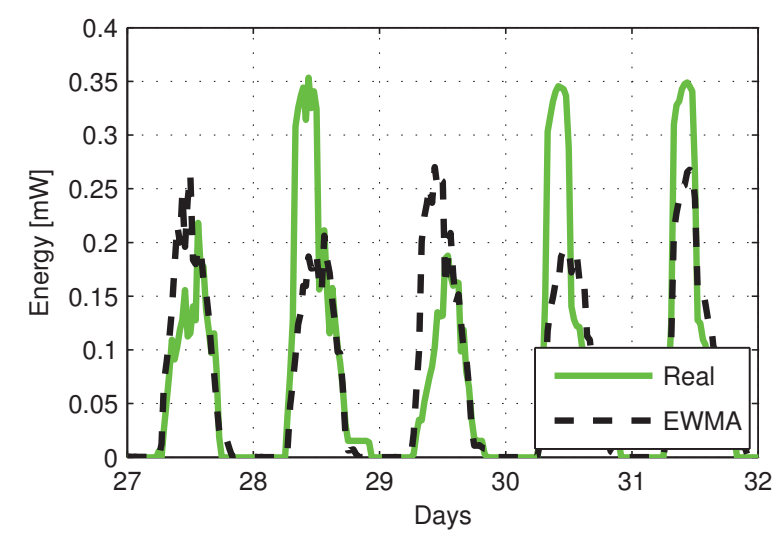

Fig. 1. EWMA energy prediction algorithm

WCMA algorithm uses an $E$ matrix of size $D \times N$ that stores $N$ energy values for each $D$ past days. Hence, $E(i, j)$ is the energy stored in the matrix for the $j^{\text {th }}$ sample on the $i^{\text {th }}$ day, and the predicted value is related to the previous sample in the same day and the mean value of the past samples (at the same hour of the day):

$$
E(d, n+1)=\alpha \cdot E(d, n)+G A P_{k} \cdot(1-\alpha) \cdot M_{D}(d, n+1)
$$

where $\alpha$ is a weighting factor similar to the EWMA algorithm, and $M_{D}(d, n+1)$ is the mean of $D$ past days at $n+1$ sample of the day:

$$
M_{D}(d, n)=\frac{\sum_{i=d-1}^{d-D} E(i, n)}{D}
$$

The main innovation in our algorithm is the inclusion of the factor $G A P_{k}$. This factor measures the solar conditions in the present day relative to the previous days. To compute the $G A P_{k}$ factor, we first define a vector $\mathbf{V}=\left[v_{1}, v_{2}, \cdots, v_{K}\right]$ with $K$ elements. $V$ contains the quotient of the past $K$ samples and the average solar energy available during the previous $D$ days for those samples. Therefore, a value greater that one means that today's value is larger than the mean, which represents a sunny day, and values smaller that one represents cloudy days:

$$
v_{k}=\frac{E(d, n-K+k-1)}{M_{D}(d, n-K+k-1)}
$$

Then, in order to give more importance to the closest values on time, we weight these values with the distance to the actual point in time using vector $\mathbf{P}=\left[p_{1}, p_{2}, \cdots, p_{K}\right]$ as follows:

$$
p_{k}=\frac{k}{K}
$$

Finally, the weighting factor, $G A P_{k}$, is computed:

$$
G A P_{k}=\frac{\mathbf{V} \cdot \mathbf{P}}{\sum \mathbf{P}}
$$


TABLE I

SOLAR ENERGY PREDICTION EXAMPLE

\begin{tabular}{ccccc}
\hline \hline \multicolumn{5}{c}{ Solar Panel energy evolution in $\mathrm{mW}$} \\
\hline \hline \multicolumn{1}{c}{$\mathbf{n - 2}$} & $\mathbf{n - 1}$ & $\mathbf{n}$ & $\mathbf{n + 1}$ \\
\hline $\mathbf{d - 4}$ & 277 & 272 & 221 & 263 \\
\hline $\mathbf{d - 3}$ & 350 & 353 & 347 & 347 \\
\hline $\mathbf{d - 2}$ & 345 & 346 & 349 & 353 \\
\hline $\mathbf{d - 1}$ & 249 & 255 & 314 & 289 \\
\hline $\mathbf{d}$ & 342 & 256 & 230 & $? ? ?$ \\
\hline Mean & 305 & 306 & 307 & 313 \\
\hline $\mathbf{V}$ & 1.12 & 0.84 & 0.75 & \\
\hline $\mathbf{P}$ & 0.33 & 0.67 & 1.00 & \\
\hline
\end{tabular}

Example: Table I shows an example of how WCMA computes the $G A P_{k}$ factor for the next predicted value $E(d, n+1)$ with $D=4, K=3$. The Mean vector contains the mean value of the previous four days, $V$ has the quotient of the elements in row d divided by the Mean (element by element), and $P$ is the weighting factor for $V$. Finally, the $G A P_{k}$ value is defined as follows:

$$
G A P_{k}=\frac{(1.12,0.83,0.75) \times(0.33,0.67,1.00)}{\sum(0.33,0.67,1)}
$$

And the predicted value with $\alpha=0.7$ is:

$$
E(d, n+1)=0.7 \cdot 230+0.84(1-0.7) \cdot 313
$$

\section{A. WCMA's parameters optimization}

To optimize all the needed parameters in WCMA, i.e., the size of the $E$ matrix, $(D \times N), \alpha$ factor and number $K$ of past samples to weight, we must define the error function to evaluate and relevant constraints. To optimize these values we have recorded the energy available from the solar panel [13] every minute during 45 consecutive days. As we want to predict the sun evolution, the night values will be discarded in the computation of the error. We consider night values all the samples with less than $10 \%$ of the maximum. The error function for a record of $N$ points is given at the percentage:

$$
E r r=\frac{1}{N} \sum_{i=1}^{N} a b s\left(1-\frac{E_{\text {Real }}}{E_{\text {Pred }}}\right)
$$

where $E_{\text {Real }}$ denotes the real energy value and $E_{\text {Pred }}$ is the estimated value.

To optimize the predictor performance, not only focus on minimizing the error, but we also try to ensure all possible tradeoffs between accuracy and duty cycle are met. When more samples are collected per day, the estimate of the next value is more precise at the cost of frequent sensor node wakeups which can lead to a negative impact on the overall energy consumption. On the other hand, a too low sampling rate does not give the sufficient data to WCMA to estimate the energy harvesting rate, which would make the sensor node calibration difficult. Based on our experiments performed on
Error with $\mathrm{K}=6 \mathrm{Min}=10.5 \%$

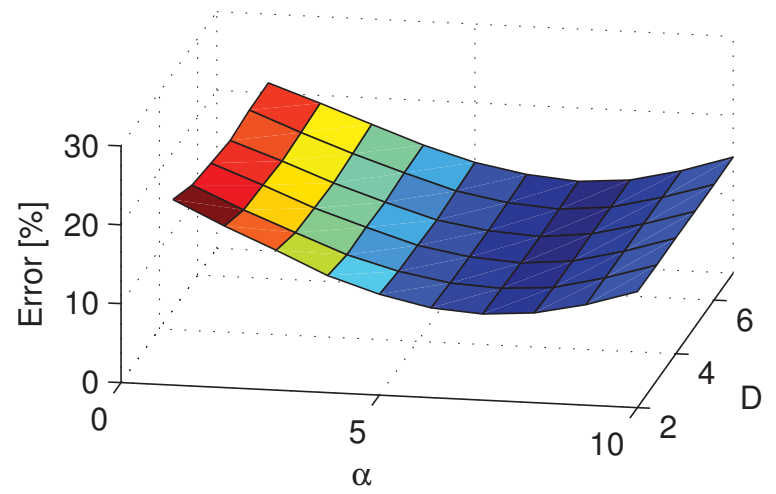

Fig. 2. Estimated error for $N=48$ and $K=6$

Error with $\alpha=7$ Min=9.8\%

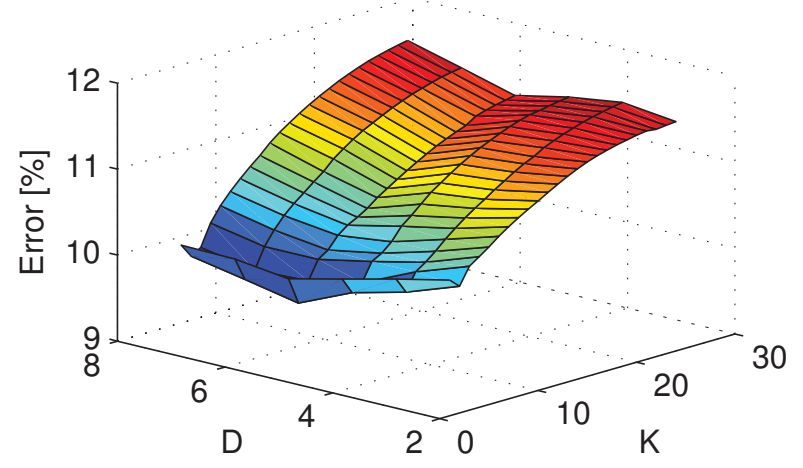

Fig. 3. Estimated error for $\mathrm{N}=48$ and $\alpha=7$

real sensor nodes ([6]), a sample period of 30 minutes (i.e., 48 samples/day) gives a reasonably accurate prediction with a low duty cycle and a small memory footprint. Figure 2 shows the estimated error of the prediction as a function of the weighting factor, and the number of days $\mathrm{D}$, for a fixed number of past values $K=6$ and samples per day $N=48$. Selecting a weighting factor $(\alpha)$ of 0.7 gives a minimal error, independent of the number of past days stored in the matrix. Hence, we will use the value of 0.7 in our optimization process of WCMA.

Figure 3 shows the prediction error versus $D$ and $K$, with $\alpha$ and $N$ fixed. Our experiments indicate that if the number of past samples $K$ is above 5, then the error quickly increases because it takes into account too many samples of the weather pattern of each day. Since the number of past days does not influence the error as much as the number of past samples $K$ for a particular day, then we can use fewer days for the estimate, which lowers the computational cost of WCMA without a significant accuracy loss. As a result of our analysis, WCMA model used in this paper has the following parameters to minimize its prediction error: $D=4$ days, $N=48$ samples/day, $K=3$ past samples, and $\alpha=0.7$. 


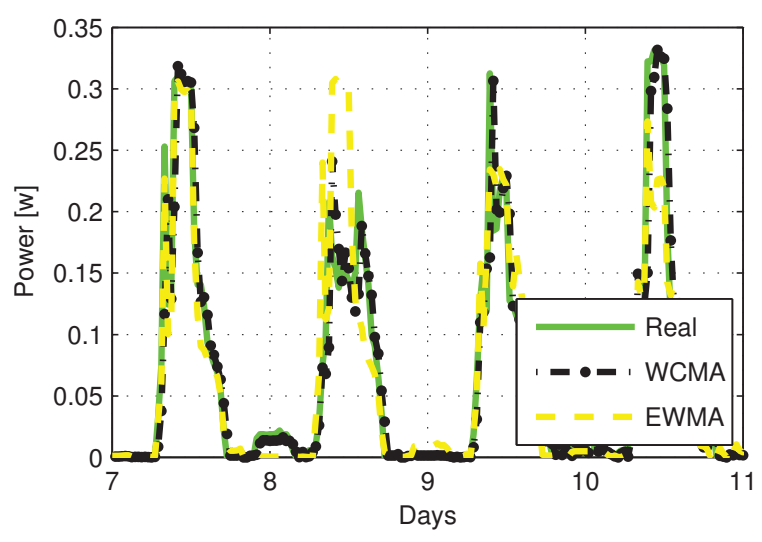

Fig. 4. Prediction accuracy of WCMA vs. EWMA

\section{B. Comparison of WCMA vs. EWMA}

We compare the energy prediction accuracy of WCMA to EWMA. Figure 4 shows four consecutive days of different weather conditions, and predicted values using both algorithms. The first and third days correspond to sunny conditions and the second and the fourth are cloudy. Since EWMA only uses values from previous days at the exact same time period, if the weather conditions change from one day to another, this method has a large error in prediction (i.e., close to $30 \%$ ). On the other hand, WCMA produces a much better results because it uses the values from the same hour over a number of previous days and the past values from the same day, which help calibrate against the actual weather conditions. Overall, EWMA gives an average error of $28.6 \%$ compared to $9.8 \%$ obtained by our new algorithm WCMA, over all 45 days of the collected solar panel data.

\section{EXPERIMENTAL SETUP AND RESUlts}

We assess the performance of WCMA in a real-life energy harvested wireless sensor node, i.e., the Shimmer platform [6], which is an active sensing platform for structural health monitoring. Shimmer uses a super capacitor as energy storage unit and solar panel as energy harvesting unit. It is able to perform active sensing using a matrix of 16 piezoelectric actuators/sensors (PZTs) to find structural damage. It launches a wave signal via one PZT through the material and samples the resulting wave via another PZT. Then Shimmer uses the acquired signal to perform complex processing on its DSP to determine if the structure is damaged or not. Shimmer is also equipped with a ZigBee-compatible radio link. Thus, it can send the sampled data or the results through its on-board processing to a remote server. The types of tasks that can be executed on the Shimmer platform are:

- Actuate/Sample: Shimmer has 16 PZTs which result in 240 different paths that can be tested. This task is characterized by a mean power consumption of $1027 \mathrm{~mW}$ and an execution time of $1.1 \mathrm{~ms}$, producing $20 \mathrm{Kbytes}$ of data.
TABLE II

SOLAR ENERGY PREDICTIION RESULTS

\begin{tabular}{ccccc}
\hline \hline Day & $\mathrm{E}_{\text {real }} \mathrm{J}$ & Algorithm & EJ & Err\% \\
\hline \hline \multirow{2}{*}{7} & \multirow{2}{*}{571.72} & WCMA & 550.44 & 3.72 \\
\cline { 3 - 5 } & & EWMA & 535.50 & 6.34 \\
\hline \multirow{2}{*}{8} & \multirow{2}{*}{284.63} & WCMA & 255.60 & 10.20 \\
\cline { 3 - 5 } & & EWMA & 543.60 & -90.99 \\
\hline \multirow{2}{*}{9} & \multirow{2}{*}{400.61} & WCMA & 360.00 & 10.14 \\
\cline { 3 - 5 } & & EWMA & 423.00 & -5.59 \\
\hline \multirow{2}{*}{10} & \multirow{2}{*}{609.50} & WCMA & 597.60 & 1.95 \\
\cline { 3 - 5 } & & EWMA & 406.80 & 33.26 \\
\hline
\end{tabular}

- Process: Different algorithms can be employed to process the sampled data; they can vary from simple time domain pattern matching to complex filtering and frequency domain analysis. Thus, this task has a mean power consumption of $680 \mathrm{~mW}$ and an execution time of $55 \mathrm{~ms}$ (light process) or $3470 \mathrm{~ms}$ (complex process).

- Send: When no damage is detected, little data needs to be sent over the radio, but in other situations the whole data record gathered is necessary to perform an analysis of the damage evolution over long periods. This task has a power consumption of $165 \mathrm{~mW}$ and requires $6 \mathrm{~ms}$ to send a packet of up to 255B. Shimmer is usually placed in hard-to-reach locations, with limited wireless connectivity. It needs to execute the damage detection process daily. Thus, an energy estimator is mandatory to carefully choose when the damage assessment should be performed, and what kind of processing can be done with the currently available energy and likely to be harvested in near future. Table II shows the values of predicted and actual energy available at noon during a period of $30 \mathrm{~min}$ for the time record depicted in Figure 4. We focus on these specific times to illustrate the difference between EWMA and WCMA when it comes to intraday prediction.

As Table I shows, WCMA has a maximum energy prediction error of only $10 \%$, while EWMA can have errors of up to $90 \%$. This large EWMA energy estimation error can cause incorrect energy management decisions by Shimmer. Shimmer needs to Actuate/Sample, perform full Processing and Send the data obtained from all 240 PZT paths in order to identify and localize damage in the structure. When there isn't enough energy to perform this whole analysis, the complexity of processing task has to be reduced to ensure that at least some feedback is obtained on the current structural health. Thus, in the following experiments we evaluate how well WCMA and EWMA perform as energy availability estimators in situations where energy resources are at a premium and not all tasks can be executed.

Checking the whole structure means that all 240 different paths are actuated/sensed, with the total energy cost of $240 * 1.2 \mathrm{~mJ}=0.27 \mathrm{~J}$. Sending all this processed data requires $240 * 77.6 \mathrm{~mJ}=18.6 \mathrm{~J}$. The rest of the available energy can be 
used to perform processing by the DSP. While the goal is to run full processing at $3.47 \mathrm{~s}$ per path each day, when there is not enough energy available, then a fraction of paths run only light processing, resulting in the overall lower average processing time per path. We next report this DSP time per path assuming the perfect prediction of energy harvesting capabilities (oracle), or when using either WCMA or EWMA to predict the amount of energy harvested over the next 30min period. To illustrate our ideas we focus on the $30 \mathrm{~min}$ period around noon each of the days outlined in Table II.

- Day 7: Both WCMA and EWMA do a great job predicting for consistently sunny days resulting in the average DSP time per path of $3.26 \mathrm{~s}$ and $3.17 \mathrm{~s}$ respectively, compared to $3.39 \mathrm{~s}$ by oracle predictor.

- Day 8: When a sudden change in weather occurs, WCMA is able to adapt quickly resulting in much better processing allocation relative to EWMA. Average DSP per path time for $\mathrm{WCMA}=1.45 \mathrm{~s}$, while oracle is only slightly higher at $1.63 \mathrm{~s}$. In contrast, EWMA significantly overestimates the energy entering the system thus scheduling too many full processing tasks with average DSP time of $3.22 \mathrm{~s}$. As a result, Shimmer runs out of energy before finishing the whole structure scan, resulting in a significantly worse result.

- Day 9: Both predictors offer a good approximation of the DSP time per path. Oracle comes in at $2.34 \mathrm{~s}$ per path, WCMA: 2.09s, EWMA: 2.48s.

- Day 10: EWMA underestimates the DSP time since weather got better in day; oracle got 3.62s, WCMA: 3.55s, EWMA: 2.38s. As a result, much less processing is done if EWMA is used for prediction, leading to suboptimal results relative to WCMA.

Overall, WCMA correctly predicts all the variations of harvested energy so that Shimmer can fully utilize it to estimate the structural damage, while EWMA has significant difficulties during variable weather conditions.

\section{CONCLUSION}

In this paper we presented Weather-Conditioned Moving Average (WCMA), a new solar energy prediction algorithm with very low computational overhead. WCMA is able to effectively take into account both the current and past-days weather conditions. Thus, it reduces the error of the predicted value down to only $10 \%$ from $90 \%$ by EWMA during highly variable weather patterns. Finally, we showed on the example of Shimmer platform that correct prediction can have a significant impact on the quality of structural health monitoring results at run time.

\section{ACKNOWLEDGEMENTS}

This work is partially supported by the Spanish Government Research Grants TIN2008-00508 and CSD00C-07-20811.

\section{REFERENCES}

[1] Crossbow. Mica motes. [Online]. Available: http://www.xbow.com/Home/wHomePage.aspx

[2] V. Raghunathan, C. Schurgers, S. Park, and M. Srivastava, "Energyaware wireless microsensor networks," IEEE Signal Processing Magazine, vol. 19, no. 2, pp. 40-50, Mar. 2002.

[3] R. Pon, M. Batalin, J. Gordon, A. Kansal, D. Liu, M. Rahimi, L. Shirachi, Y. Yu, M. Hansen, W. Kaiser, M. Srivastava, G. Sukhatme, and D. Estrin, "Networked infomechanical systems: a mobile embedded networked sensor platform," in Proc. in Sensor Networks (IPSN'05), Apr. 2005, pp. 376-381.

[4] G. Sibley, M. Rahimi, and G. Sukhatme, "Robomote: A tiny mobile robot platform for large-scale ad-hoc sensor networks," in Proc. of the IEEE International Conference on Robotics and Automation (ICRA'O2), vol. 2, May 2002, pp. 1143-1148.

[5] V. Raghunathan, A. Kansal, J. Hsu, J. Friedman, and M. Srivastava, "Design considerations for solar energy harvesting wireless embedded systems," in Proc. in Sensor Networks (IPSN'05), Apr. 2005, pp. 457 462.

[6] D. Musiani, K. Lin, and T. Rosing, "Active sensing platform for wireless structural health monitoring," in Proc. in Sensor Networks (IPSN'07), Apr. 2007, pp. 390-399.

[7] D. R. Cox, "Prediction by exponentially weighted moving averages and related methods," Royal Statistical Society, vol. 23, no. 2, pp. 414-422, 1961.

[8] J. S. Hunter, "The exponentially weighted moving average," Quality Technology, vol. 18, no. 4, pp. 203-207, 1986.

[9] C. Vigorito, D. Ganesan, and A. Barto, "Adaptive control of duty cycling in energy-harvesting wireless sensor networks," in IEEE Conf. in Sensor Mesh and Ad Hoc Communications and Networks (SECON'07), 2007, pp. 21-30.

[10] A. Kansal, J. Hsu, S. Zahedi, and M. B. Srivastava, "Power management in energy harvesting sensor networks," in ACM Transactions on Embedded Computing Systems (TECS'07), vol. 6, no. 4, Sep. 2007.

[11] H. Suehrcke and P. McCormick, "A performance prediction method for solar energy systems," Solar energy, vol. 48, no. 3, pp. 169-175, 1992.

[12] R. Iqdour and A. Zeroual, "Prediction of daily global solar radiation using fuzzy systems," Int. Journal of Sustainable Energy, vol. 26, no. 1, pp. 19-29, 2007.

[13] PowerFilm. Oem components. [Online]. Available: http://www.powerfilmsolar.com/ 